\title{
Determining Chilling Injury Induction in Green Peppers Using Nondestructive Pulse Amplitude Modulated (PAM) Fluorometry
}

\author{
Susan Lurie, Reuven Ronen, and Shimon Meier \\ Department of Fruit and Vegetable Storage, Volcani Center, Agricultural Research Organization, Bet \\ Dagan 50250, Israel \\ Additional index words. Capsicum annuum, leakage, low-temperature injury
}

\begin{abstract}
Storing 'Maor' green bell peppers (Capsicum annuum L.) for 3 weeks at $2 \mathrm{C}$ resulted in the development of chilling injury (CI) evidenced as surface pitting. Fruit held at $8 \mathrm{C}$ did not develop any CI symptoms, but, after 3 weeks of storage, the fruit began to change color from green to red. PAM fluorometry was used to measure changes in photosynthetic competency in whole green bell peppers. Three photosynthetic characteristics could be measured by this method: quantum yield $(\mathbf{F m} / \mathbf{F e})$, photochemical quenching $(\mathrm{Qp})$, and nonphotochemical quenching (Qnp). Fm/Fo decreased $90 \%$ during the first week of storage at 2C and remained low thereafter, while Qnp decreased after 2 weeks at 2C, just before the peppers began to develop CL Qp was similar at both storage temperatures. Potassium leakage as a CI measurement also increased in excised pepper discs after 2 weeks at $2 \mathrm{C}$. The results indicate that PAM fluorometry can measure CI nondestructively before tissue damage is visible in green peppers.
\end{abstract}

Remarkable progress has been made recently in the understanding and practical use of chlorophyll fluorescence in plant science, spurred by the need for quantitative, noninvasive, rapid methods to assess photosynthesis in intact leaves (van Kooten and Snel, 1990). In addition, the potential of chlorophyll fluorescence as a naturally occurring internal probe sensitive to stress-related cellular injury has been investigated (Smillie and Hetherington, 1983). When illuminated, chlorophyll emits a red fluorescence, of which part-the variable fluorescence-is responsive to changes in photosystem II activity (Papageorgiou, 1975). Therefore, arty stress, which directly or indirectly affects photosynthetic metabolism, is likely to change the yield of this fluorescence when applied to green plant tissue.

Most studies of the effects of environmental stress on fluorescence have measured only from the initial $(\mathrm{Fe})$ to the maximal (Fro) level, i.e., quantum yield (Fm/Fe), whether on leaves or fruit (Abbott et al., 1987; Smillie and Hetherington, 1983; Walker et al., 1990). PAM fluorometry measures additional characteristics of photosynthesis. In addition to measuring Fm/Fo, this technique enables the researcher to determine features of photochemical quenching (Qp), due to electron transport, and nonphotochemical quenching (Qnp), the proton pumping of ATPase and the $\mathrm{pH}$ gradient across the thylakoid membrane. Thus, this procedure gives more information about the state of the photosynthetic units than that derived from normal fluorescence measurements (Schreiber, 1986). We have used PAM fluorometry to follow the development of chilling injury (CI) in whole green peppers, correlating it with $\mathrm{K}$ leakage from fruit discs and the development of visible CI symptoms.

\section{Materials and Methods}

Field-harvested 'Maor' green peppers were placed directly in storage at either 8 or $2 \mathrm{C}$ at $90 \%$ relative humidity (RI-I). At harvest

\footnotetext{
Received for publication 9 Dec. 1992. Accepted for publication 29 May 1993. We thank Shmuel Malkin for the use of his PAM fluorometer. This work was supported by a U.S.-Israel binational agricultural research and development grant. This is contribution no. 3674-E, 1992 series from the Volcani Center. The cost of publishing this paper was defrayed in part by the payment of page charges. Under postal regulations, this paper therefore must be hereby marked advertisement solely to indicate this fact.
}

and after each week of storage for 4 weeks, peppers were placed at 20C, $85 \%$ RH, for 5 days. At each observation time, three replications of 10 fruit from each storage temperature were examined for color changes and CI, as evidenced by surface pitting. Five fruit from each treatment were examined by PAM fluorometry. The fruit were brought to $20 \mathrm{C}$ and dark-adapted for at least $20 \mathrm{~min}$ before being measured. Initially, the warming of fruit removed from 2 or $8 \mathrm{C}$ was followed by a thermistor in the pericarp; $2 \mathrm{~h}$ was sufficient to bring the peppers to 20C. Discs were prepared from the same five fruit after PAM measurement and incubated for $\mathrm{K}^{+}$ leakage.

PAM fluorometry. Fluorescence was measured using a PAM fluorometer (Walz, Effeltrich, Germany) equipped with a fiberoptic probe (1 cm in diameter) that was placed on the fruit surface. Depending on light conditions, four fluorescent states could be distinguished (Fig. 1), with Qp and Qnp varying between 0 and 1. In dark-adapted tissue, exposing fruit to a weak modulating measuring light of $1.6 \mathrm{kHz}$ at $650 \mathrm{~nm}$ activated Fo in state 1 . In this state, all photosystem II reaction centers were open while the photosynthetic membrane was in the nonenergized state $(\mathrm{Qp}=1$ and Qnp =0). Then a saturating light pulse of $7400 \mu \mathrm{mol} \cdot \mathrm{m}^{-2} \cdot \mathrm{s}^{-1}$ for $600 \mathrm{msec}$ was given to excite Fm and move the system into state 2 . In this state, all photosystem II reaction centers were closed (Qp $=0$ ) while the photosynthetic membrane was still in a nonactivated state $(\mathrm{Qnp}=0)$. After the saturating pulse, an actinic red light of 75 $\mu \mathrm{mol} \cdot \mathrm{m}^{-2} \cdot \mathrm{s}^{-1}$ of $650 \mathrm{~nm}$ was turned on, while pulses of saturating light continued to be given every $5 \mathrm{sec}$. The system oscillated between state 3 (actinic light) and state 4 (actinic light plus saturating pulse). Qp and Qnp designate the relative decrease in variable fluorescence yield due to photosynthetic and nonphotosynthetic processes, respectively, derived from van Kooten and Snel (1990).

$$
\begin{aligned}
\mathrm{Qp} & =\mathrm{F}^{\prime} \mathrm{m}-\mathrm{F} / \mathrm{F}^{\prime}-\mathrm{m}-\mathrm{Fo} \\
\mathrm{Qnp} & =\mathrm{Fm}-\mathrm{F}^{\prime} \mathrm{m} / \mathrm{Fm}-\mathrm{Fo}
\end{aligned}
$$

Potassium leakage. Two 1-cm discs from each of five peppers were cut by a cork borer and each pair was incubated in $5 \mathrm{ml}$ of 0.35 $\mathrm{M}$ sorbitol. At the end of $3 \mathrm{~h}$, the discs were removed from solution, 


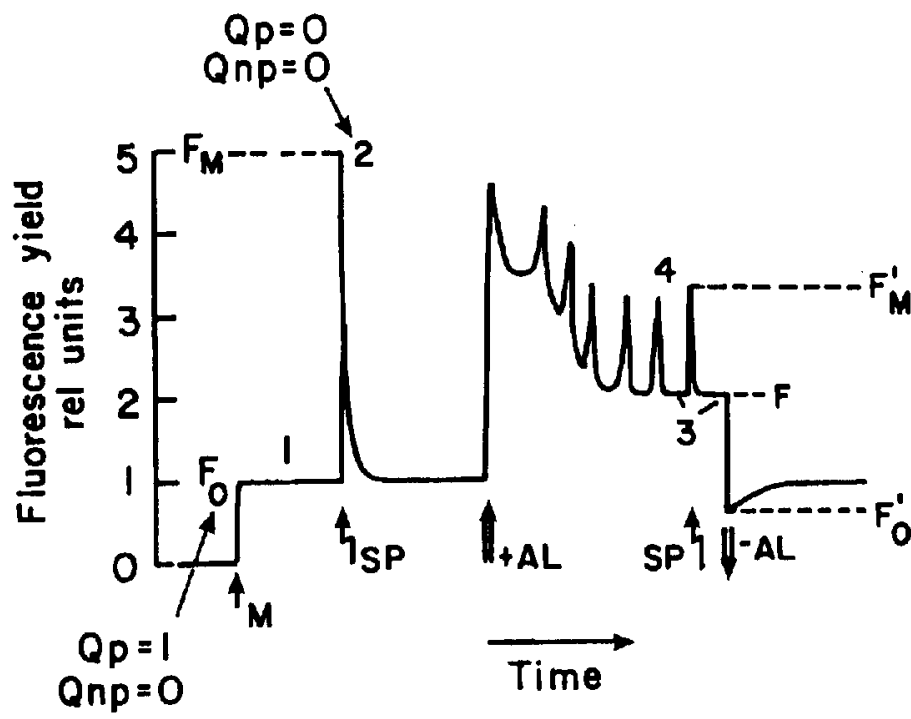

Fig. 1. Fluorescence yield and and quenching analysis as measured by pulse amplitude modulation fluorometry. A weak modulated measuring light(M) gives abase level fluorescence $(\mathrm{Fe})$. This is state 1 fluorescence, where all photosystem II reaction centers are open. Then a saturating light pulse (SP) is given to close the photosystem II reaction sites (state 2), and maximal fluorescence (Fro) is measured, A continuous actinic light (AL) is turned on and saturating light pulses continue to be given, thus alternating the system between states $3\left(F^{\prime} \mathrm{m}\right)$ and $4(\mathrm{~F})$. (Adapted from van Kooten and Snel, 1990).

frozen, thawed, and boiled for $20 \mathrm{~min}$. The incubating solution and the boiled tissue were examined for $\mathrm{K}^{+}$using a flame photometer, and the $\mathrm{K}^{+}$present in the solution after $3 \mathrm{~h}$ was expressed as percentage of total $\mathrm{K}+$.

\section{Results}

Fm/Fo of the peppers stored at $2 \mathrm{C}$ was almost 10 -fold lower than that of peppers held at $8 \mathrm{C}$ after 1 week of storage and remained low during storage (Fig. 2). When peppers were removed from $2 \mathrm{C}$ at weekly intervals and then held at 20C for 5 days, Fm/Fo after shelf life increased 4-fold over that of storage. After 1 week at $2 \mathrm{C}$, $\mathrm{Fm} / \mathrm{Fo}$ recovered to $65 \%$ and after 2 weeks to $45 \%$ of that of peppers stored at $8 \mathrm{C}$. CI symptoms appeared after 3 weeks at $2 \mathrm{C}$ (Fig. 3, top), while no CI developed on peppers stored at $8 \mathrm{C}$. Peppers stored at $8 \mathrm{C}$ had a decreased Fm/Fo after 3 and 4 weeks of storage. This decrease corresponded to chlorophyll disappearance as the peppers continued to mature at this temperature and began to change color (Fig. 3, bottom). Peppers held at $2 \mathrm{C}$ showed no color change even when moved to 20C.

Qp decreased gradually in peppers stored at 8 or $2 \mathrm{C}$, with no significant difference between the two storage temperatures (Fig. 4 ). There was no change in this coefficient during holding at $20 \mathrm{C}$. In contrast, Qnp was lower in fruit stored at $2 \mathrm{C}$ than in those at $8 \mathrm{C}$ for 2 weeks or longer (Fig. 5). This characteristic increased when the fruit were removed from 8 and then held at 20C for 5 days. In peppers moved from 2 to $20 \mathrm{C}$, Qnp recovered after 1 week of storage, but not after 2 or more weeks.

Potassium leakage from discs of freshly harvested peppers was $20 \%$ after $3 \mathrm{~h}$ of incubation (Fig. 6). Leakage increased as the fruit were stored longer at either 8 or $2 \mathrm{C}$. Peppers stored at $8 \mathrm{C}$ had increased leakage during storage. Fruit at $2 \mathrm{C}$ had a dramatic rise in leakage between 1 and 2 weeks of storage. By 4 weeks, there was a decline in leakage from discs of fruit stored at $2 \mathrm{C}$ so that leakage from fruit at both storage temperatures was similar.

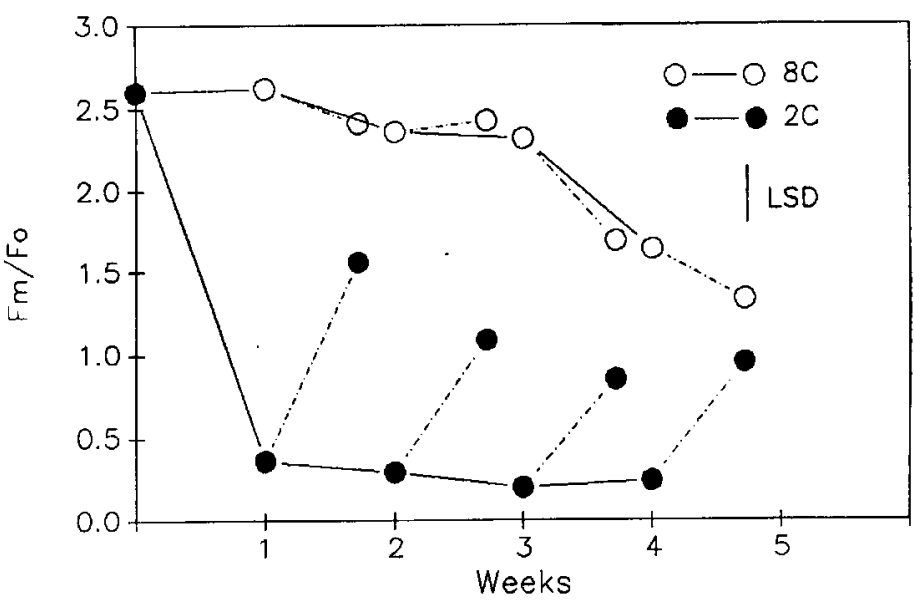

Fig, 2. The change in Fm/Fo of peppers stored for 1 to 4 weeks at 8 or $2 \mathrm{C}$ and then held for 5 days at 20C. Transfer from storage to $20 \mathrm{C}$ each week is shown by a dotted line. $\mathrm{LSD}_{0.05}$ is indicated.
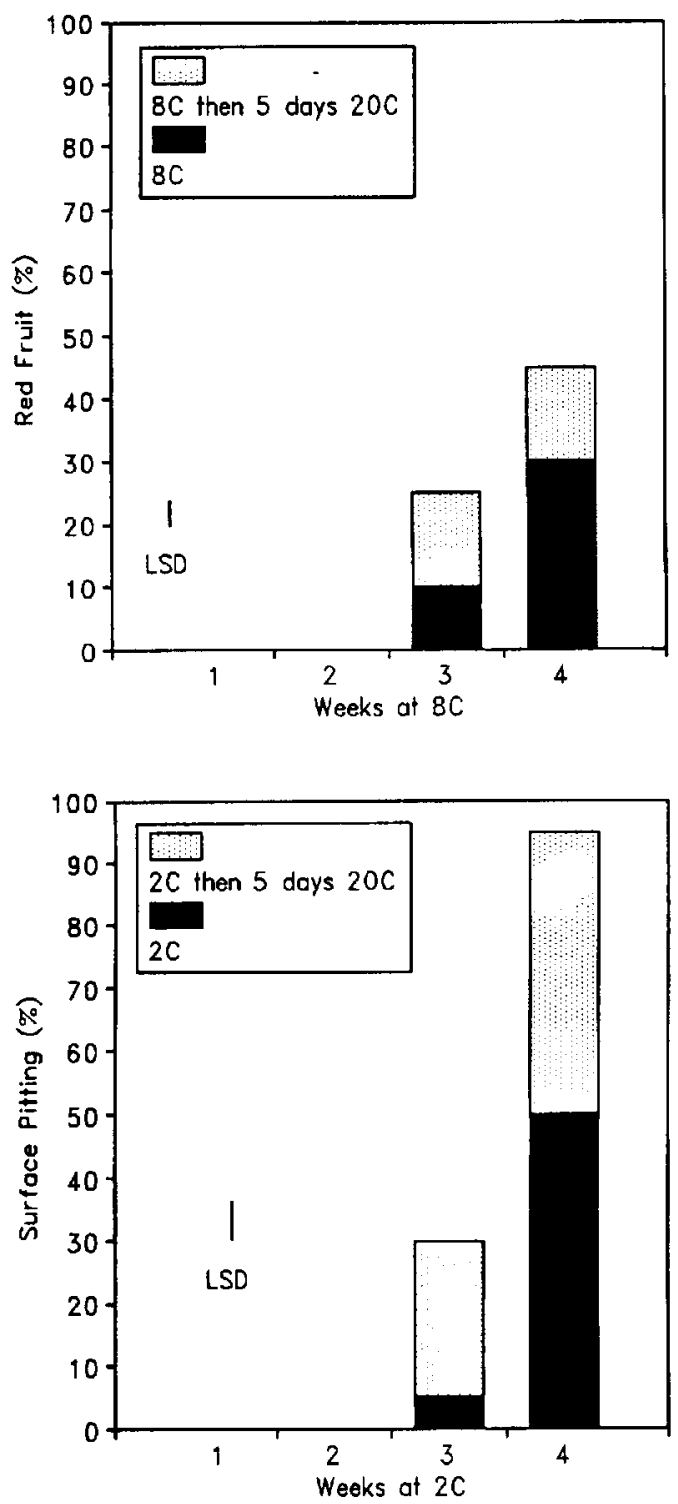

Fig.3. (A) Surface pitting in green peppers held at $2 \mathrm{C}$ for 1 to 4 weeks and then at $20 \mathrm{C}$ for 5 days. (B) Color change in green peppers held at $8 \mathrm{C}$ for 1 to 4 weeks and then at $20 \mathrm{C}$ for 5 days. $\mathrm{LSD}_{005}$ is indicated. 


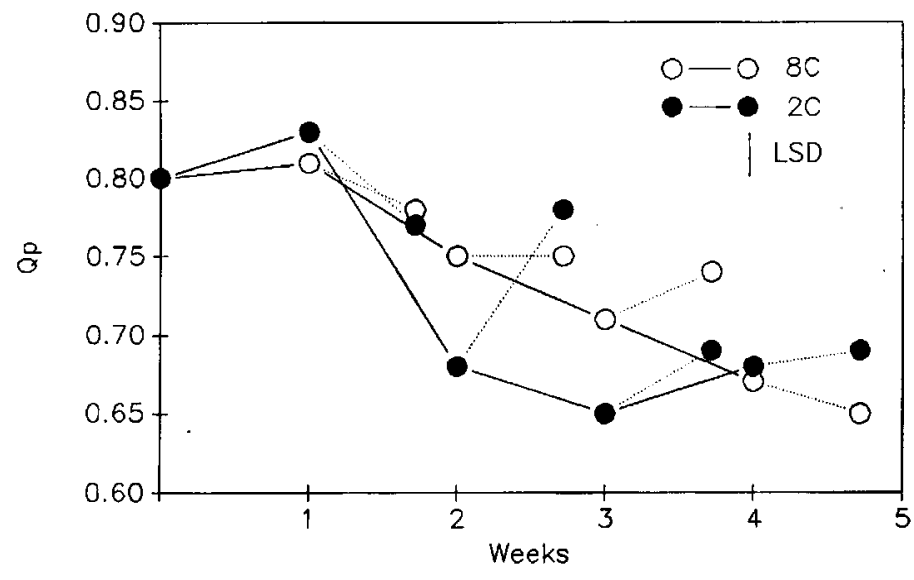

Fig. 4. The changes in Qp of peppers during storage and after 5 days at 20C following storage for 1 to 4 weeks at 2 or $8 \mathrm{C}$, Transfer from storage to $20 \mathrm{C}$ each week is shown by a dotted line. $\operatorname{LSD}_{0.05}$ is indicated.

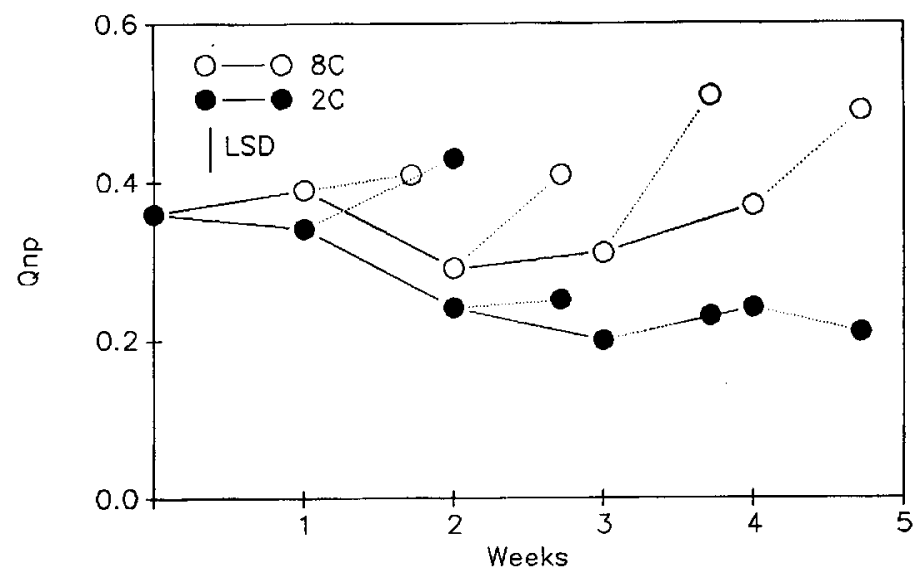

Fig. 5. The changes in Qnp of peppers during storage and after 5 days at 20C following storage for 1 to 4 weeks at 2 or $8 \mathrm{C}$. Transfer from storage to $20 \mathrm{C}$ each week is shown by a dotted line. $\mathrm{LSD}_{0.05}$ is indicated.

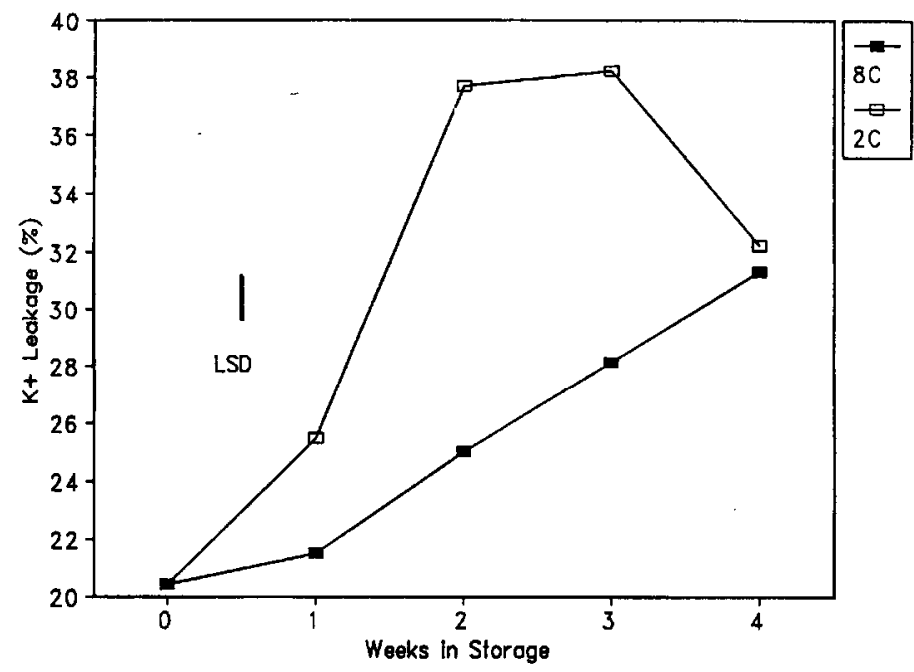

Fig. 6. Potassium leakage from discs of peppers stored at 8 or $2 \mathrm{C}$ for 1 to 4 weeks. $\mathrm{LSD}_{005}$ is indicated.

\section{Discussion}

Fm/Fo and Qnp decreased in green peppers stored at $2 \mathrm{C}$ before the appearance of visible CI signs. However, Fm/Fo responded rapidly to low temperature, even before the tissue became susceptible to CI, whereas Qnp decreased significantly only after 2 weeks at $2 \mathrm{C}$, concomitant with the increase in $\mathrm{K}^{+}$leakage from fruit discs. Moreover, during storage, Fm/Fo recovered when the fruit were transferred to 20C, while Qnp recovered after 1 week at 2C but not thereafter. Therefore, the response of Qnp to low temperature correlated more closely than $\mathrm{Fm} / \mathrm{Fo}$ to the visible CI symptoms and other physiological expressions of injury, such as leakage.

Leakage increased when plant tissues develop CI (Lafuente et al., 1991; Lurie and Klein, 1991) and has been used to indicate its development (Lafuente et al., 1991). In this study, there was a good correlation between increased $\mathrm{K}^{+}$leakage and $\mathrm{CI}$ development on the peppers after 3 weeks of storage. However, for some fruit sensitive to low-temperature storage, leakage either does not increase (Fuchs et al., 1989) or increases only after CI has developed (McCollum and McDonald, 1991) and, therefore, is of limited value as an early CI indicator. Moreover, leakage is a destructive measurement, while PAM is nondestructive and can measure many fruit in a short time.

Severe CI produces visible symptoms, but, before these are evident, changes in cell metabolism can be detected, including altered physical properties of cell membranes, anomalous respiratory activity, and electrolyte movement across membranes (Lyons, 1973). In addition, photosynthesis is affected in chillingsensitive plants. Inhibition develops on the photooxidizing side of photosystem II (Margulies and Jagendorf, 1960; Smillie and Nott, 1979; Terashima et al., 1989) and, therefore, results in a decreased Fm/Fo (Papageorgiou, 1975), as was found in the peppers. This inhibition was partially reversible at early stages of low-temperature storage. The recovery of photosystem II in chilling-sensitive plants after some days at $0 \mathrm{C}$ depends on removing the plants to a higher temperature and illuminating the leaves (Kaniug et al., 1978). We did not determine whether holding peppers at $20 \mathrm{C}$ in darkness would have prevented the recovery of Fm/Fo seen after storage at $2 \mathrm{C}$.

Although the photooxidizing side of photosystem II was inhibited by low temperature and Fm/Fo was reduced in peppers held at 2C, Qp (which reflects photosynthetic electron transport) was unaffected by low-temperature storage. This response has also been found in cucumber (Cucumis sativus L.) leaves and isolated chloroplasts, in which chilling treatments did not alter electron transport rates (Peeler and Naylor, 1988; Terashima et al., 1989) Qnp, which is a measure of the energizing of the thylakoid membranes leading to ATP formation, decreased in peppers stored at 2C. Moll and Steinbach (1986) found that chilling Oryza sativa L. led to the disruption of ATP formation earlier than inhibition of the electron transport reactions of photosynthesis. In chilled cucumber leaves, electron transport rates in thylakoids were not inhibited, but thylakoids were totally permeable to protons and, therefore, uncoupled from ATP formation (Peeler and Naylor, 1988). Apparently, the chloroplasts of green peppers respond to low temperature identically to chloroplasts from chilling-sensitive leaves.

Most fluorescent measurements of plant tissue in response to environmental stress have been on leaves. Many fruit at harvest contain chloroplasts in their peel and sometimes in their flesh, and these have been shown to be active photosynthetically, although contributing only minimally to the carbon balance of the fruit (Blanke and Lenz, 1989; Smillie, 1992). For fruit that are sensitive 
to low-temperature storage, a nondestructive, rapid method of measuring CI would be useful. The results presented here demonstrate the-potential of PAM fluorometry to estimate rapidly and nondestructively the chilling tolerance of detached organs, such as chloroplast-containing fruit. With this method, each sample can be measured in a few seconds and the signals of many samples can be stored and processed by computer.

\section{Literature Cited}

Abbott, J. A., D.T. Krizek, P. Semeniuk, H.E. Moline, and R.M. Mirecki. 1987. Refreshed delayed light emission and fluorescence for detecting pretreatment effects on chilling injury of coleus. J. Amer. Soc. Hort. Sci. 112:560-565.

Blanke, M.M. and F. Lenz. 1989. Fruit photosynthesis. Plant Cell Environ. 12:31-46.

Fuchs, Y., G. Zauberman, I. Rot, and A. Weksler. 1989. Leakage and chilling injury in avocado and mango. Acta Hort. 258:303-308.

Kaniuga, Z., B. Sochanowicz, J. Zabek, and K. Krzystyniak. 1978. Photosynthetic apparatus in chilling-sensitive plants. Planta 14012 1-1 28.

Lafuente, M.T., A. Belver, M. Guye, and M. Saltveit. 1991. Effect of temperature conditioning on chilling injury of cucumber cotyledons. Plant Physiol. 95:443-449.

Lune, S. and J.D. Klein. 1991. Acquisition of low-temperature tolerance in tomatoes by exposure to high-temperature stress. J. Amer. Soc. Hort. Sci. 116:1007-1012.

Lyons, J.M. 1973. Chilling injury in plants. Annu. Rev. Plant Physiol. 24:445-466.

Margulies, M.M. and A.T. Jagendorf. 1960. Effect of cold storage of bean leaves on photosynthetic reactions of isolated chloroplasts. Arch. Biothem. Biophys. 90176-183.
McCollum, T.G. and R.E. McDonald. 1991. Electrolyte leakage, respiration and ethylene production as indices of chilling injury in grapefruit. HortScience 26:1191-1192.

Moll, B. and K. Steinbach. 1986. Chilling sensitivity in Oryza sativa: The role of protein phosphorylation in protection against photoinhibition. Plant Physiol. 80:420-423.

Papageorgiou, G. 1975. Chlorophyll fluorescence: an intrinsic probe of photosynthesis, p. 319-371. In; Govindjee (cd.). Bioenergetics of photosynthesis. Academic Press, New York.

Peeler, T.C. and A.W. Naylor. 1988. A comparison of the effects of chilling on thylakoid electron transfer in pea (Pisun sativum L.) and cucumber (Cucumis sativus L.). Plant Physiol. 86:147-151.

Schreiber, U. 1986. Detection of rapid induction kinetics with a new type of high frequency modulated chlorophyll fluorometer. Photosyn. Res. 9:261-271.

Smillie, R.M. 1992. Calvin cycle activity in fruit and the effect of heat stress. Scientia Hort. 51:83-95.

Smillie, R.M. and S.E. Hetherington. 1983. Stress tolerance and stressinduced injury in crop plants measured by chlorophyll fluorescence in vivo. Plant Physiol. 72:1043-1050.

Smillie, R.M. and R. Nott. 1979. Assay of chilling injury in wild and domestic tomatoes based on photosystem activity of chilled leaves. Plant Physiol. 63:796-801.

Terashima, T., L.-K. Huang, and C.B. Osmond. 1989. Effects of leaf chilling on thylakoid functions, measured at room temperature, in Cucumis sativus L. and Oryza sativa L. Plant Cell Physiol. 30841-850. van Kooten, O. and J.F.H. Snel. 1990. The use of chlorophyll fluorescence nomenclature in plant stress physiology. Photosyn. Res. 25: 147-1 50.

Walker, M.A., D.M. Smith, K.P. Pauls, and B.D. McKersie. 1990. A chlorophyll fluorescence screening test to evaluate chilling tolerance in tomato. HortScience 25:334-339. 